\title{
Laparoendoscopic Single-Site Nephroureterectomy with Bladder Cuff Excision for Upper Urinary Tract Transitional-Cell Carcinoma: Technical Details Based on Oncologic Principles
}

\author{
Yong Hyun Park, M.D., Sung Yul Park, M.D., Ph.D., ${ }^{2}$ and Hyeon Hoe Kim, M.D., Ph.D.
}

\begin{abstract}
Purpose: To describe our technical details of laparoendoscopic single-site (LESS) nephroureterectomy with bladder cuff excision for the management of upper urinary tract transitional-cell carcinoma (TCC) based on oncologic principles.

Patients and Methods: Two patients underwent LESS nephroureterectomy for upper urinary tract TCC. In both cases, we used a homemade single-port device that consisted of a wound retractor and a surgical glove. Using the flexible laparoscopic instruments, nephrectomy was performed using procedures similar to those of conventional laparoscopic nephrectomy. Bladder cuff excision was performed laparoscopically using the same procedure with open technique.

Results: All procedures were completed successfully without conversion to conventional laparoscopic or open surgery and without additional extraumbilical trocars or incisions. LESS nephreoureterectomy with bladder cuff excision was performed in 385 and 285 minutes with estimated blood loss of 100 and $350 \mathrm{~mL}$, respectively. Both patients were discharged on postoperative day 3 without perioperative complications.

Conclusions: LESS nephroureterectomy with bladder cuff excision for upper urinary tract TCC is a minimally invasive technique that may reproduce the open surgical technique and adhere to oncologic principles.
\end{abstract}

\section{Introduction}

$\mathbf{T}$

HE STANDARD TREATMENT for patients with upper urinary tract transitional-cell carcinoma (TCC) is nephroureterectomy with bladder cuff excision. Since it was first introduced by Clayman and associates ${ }^{1}$ in 1991, laparoscopic nephroureterectomy results in decreased postoperative pain, shorter hospital stay, more rapid convalescence, and improved cosmesis compared with open surgery. These advantages are the fundamentals of scarless surgery, and the experience and use of laparoendoscopic single-site (LESS) surgery continues to expand among urologists. ${ }^{2-4}$ Preliminary results in LESS nephroureterectomy demonstrate that the techniques are feasible with advanced skills and optimal instrumentation. ${ }^{3-5}$ The proper technique for distal ureterectomy is still an area of controversy, however.

We describe a technique for LESS nephroureterectomy with bladder cuff excision for upper urinary tract TCC that may reproduce the open surgical technique and adhere to oncologic principles.

\section{Patients and Methods}

Two patients, a 68-year-old man (body mass index [BMI] of $20.69 \mathrm{~kg} / \mathrm{m}^{2}$ ) and a 56-year-old woman (BMI of $21.4 \mathrm{~kg} / \mathrm{m}^{2}$ ) with a diagnosis of TCC of the left renal pelvis underwent transperitoneal LESS nephroureterectomy. The surgical decisions were made after obtaining informed consent from the patients and Institutional Review Board approval from the ethical committee of the hospital.

\section{Operative technique}

The patients were anesthetized generally, and then placed in a flank position with the affected side elevated 70 degrees. A 4-cm umbilical skin incision was made and extended down to the peritoneum. We constructed a homemade single-port device according to the previously described technique. ${ }^{6}$ First, a medium-sized Alexis wound retractor (Applied Medical, Rancho Santa Margarita, CA) was inserted through the incision, thus stretching the fascia enough to allow laparoscopic

\footnotetext{
${ }^{1}$ Department of Urology, Seoul National University College of Medicine, Seoul, Korea.

${ }^{2}$ Department of Urology, Hanyang University College of Medicine, Seoul, Korea.
} 


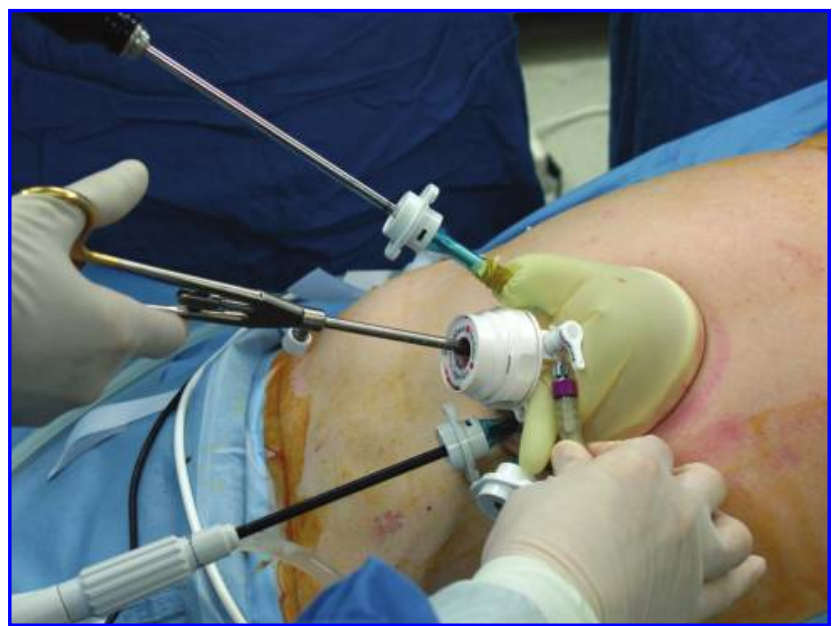

FIG. 1. Homemade single-port device used in laparoendoscopic single-site nephroureterectomy.

instruments to pass into the abdomen. A single-port device was made by attaching a size $61 / 2$ surgical glove to the opening of the port, and then securing four trocars to the fingers of the glove using rubber bands. The glove was fixed to the outer ring of the wound retractor followed by insufflation of the peritoneum with $14 \mathrm{~mm} \mathrm{Hg} \mathrm{CO}_{2}$ (Fig. 1). Possible instability was prevented by folding the lower end of the glove with Alexis wound retractor together several times to reduce the distance between the trocar and the incision.

Using flexible laparoscopic instruments (Covidien, Norwalk, CT, and CambridgeEndo, Framingham, MA), the LESS nephrectomy was performed in the same process as conventional laparoscopic nephrectomy. After the incision of the line of Toldt was made, we proceeded with the dissection medially until the hilum was exposed. The renal artery was first ligated with titanium clips, and then the renal vein was transected using Hem-o-lok clips or an endovascular stapler. After complete freeing of the kidney, the distal ureter was clipped without transection and dissected as low as possible until reaching the level of bifurcation of the common iliac artery.

After the nephrectomy was completed, we changed the position of the patients to a supine position with a 30-degree Trendelenburg decline and the affected side elevated slightly to perform the distal ureterectomy. After the ureter was retracted, the ureter was dissected caudally until the detrusor muscle fibers at the ureterovesical junction were reached, and then clipped $1 \mathrm{~cm}$ above the ureterovesical junction. Two stay stitches were placed in the bladder cuff just at the edges of the dissected detrusor muscle using a 3-0 polyglactin suture.

The intramural distal ureter was fully mobilized, the bladder was opened, and the ipsilateral ureteral orifice was taken out, including a 1-cm bladder cuff using flexible laparoscopic endoshears. The bladder opening was exposed by pulling the stay stitches and thereafter closed using a conventional rigid needle driver and dissector in one layer with 2-0 polyglactin interrupted sutures prepared with a Hem-olok clip and LapraTy clip at the terminal end (Fig. 2). After the suture was passed, additional Hem-o-lok and LapraTy clips were used to secure the suture.

Urinary leakage was examined and then excluded after filling the bladder with $200 \mathrm{~mL}$ saline. The specimen was en-

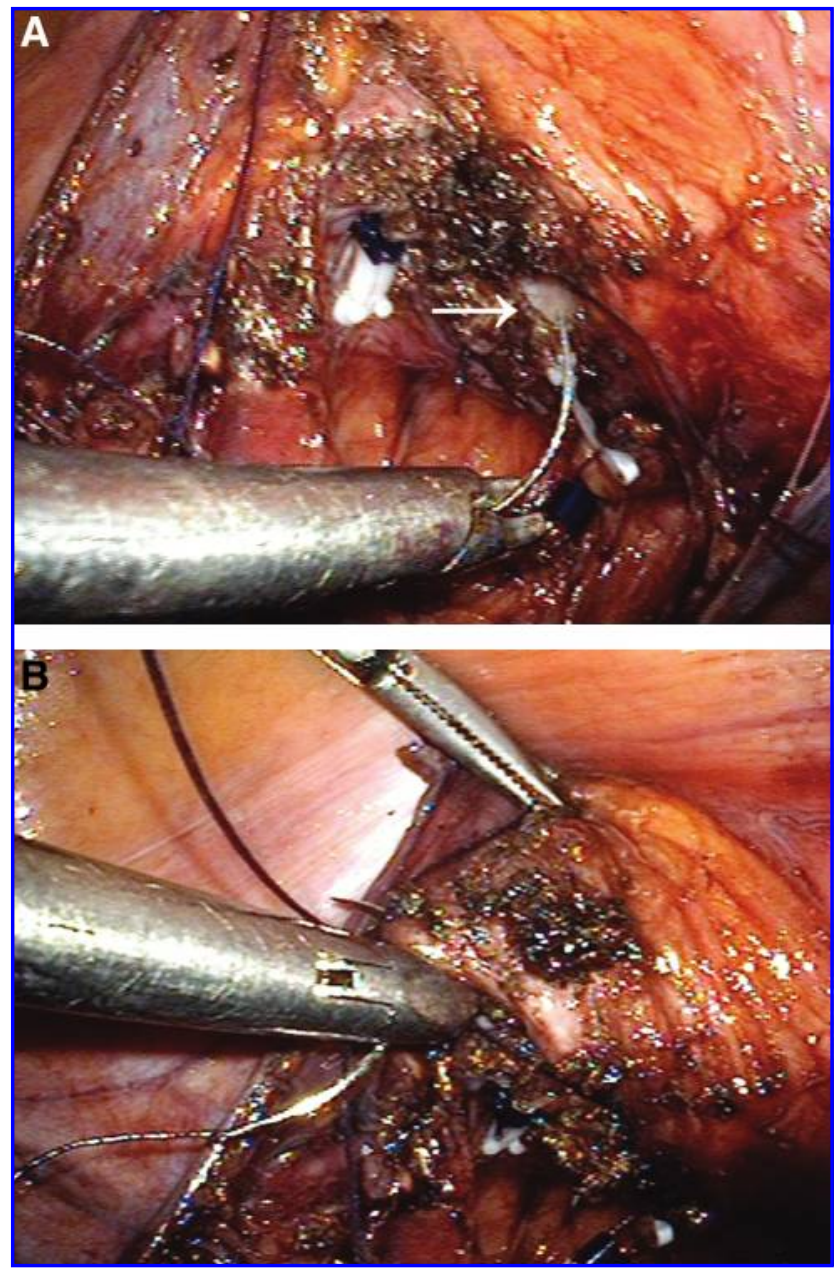

FIG. 2. The opened bladder (A, white arrow) was closed in one layer with a 2-0 polyglactin suture using a conventional rigid needle driver $(\mathbf{B})$.

trapped within the laparoscopic bag and retrieved through the umbilical incision without morcellation (Fig. 3). The incision was closed with a subcuticular absorbable suture (Fig. 4).

\section{Results}

Both LESS nephroureterectomies were completed successfully without additional extraumbilical trocars or conversion to conventional laparoscopic or open surgery. Table 1 shows the perioperative data of the patients.

The 56-year-old woman had a $1.5-\mathrm{cm}$ left renal pelvic enhancing mass. The total operative time was 385 minutes (time for nephrectomy, $145 \mathrm{~min}$; time for bladder cuff excision, $240 \mathrm{~min}$ ); estimated blood loss was $100 \mathrm{ml}$, and postoperative hospital stay was 3 days. Cystography performed 3 days after surgery showed no urine leakage; after cystography, the urethral Foley catheter was removed. Histopathologic examination revealed $\mathrm{pT}_{1}$ and grade 3 TCC with a negative surgical margin at the distal edge of the bladder cuff.

The 68-year-old man had a $4.0-\mathrm{cm}$ left renal pelvic mass with multiple small para-aortic lymph nodes. LESS nephroureterectomy with bladder cuff excision and para-aortic lymph node dissection (Fig. 5) was performed in 285 minutes (time for nephrectomy, $175 \mathrm{~min}$; time for bladder cuff excision, 

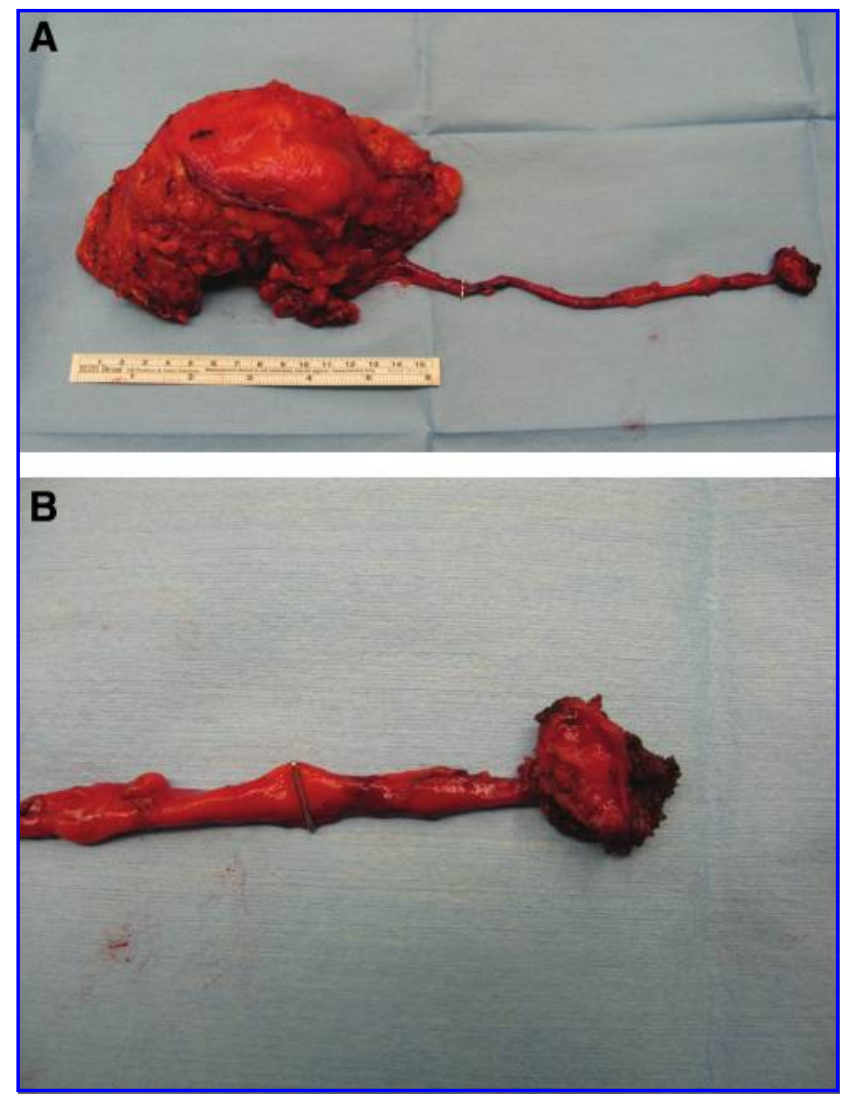

FIG. 3. The removed specimen (A) with a well-circumscribed bladder cuff (B).

$110 \mathrm{~min}$ ) with estimated blood loss of $350 \mathrm{~mL}$. In this case, the time for nephrectomy was longer than the first case because of the para-aortic lymph node dissection. The urethral Foley catheter was removed on postoperative day 7 after cystography confirmed absence of urine leakage. Histopathologic examination revealed a negative margin $\mathrm{pT}_{3}$ and grade $3 \mathrm{TCC}$ with no lymph node metastasis in eight lymph nodes retrieved.

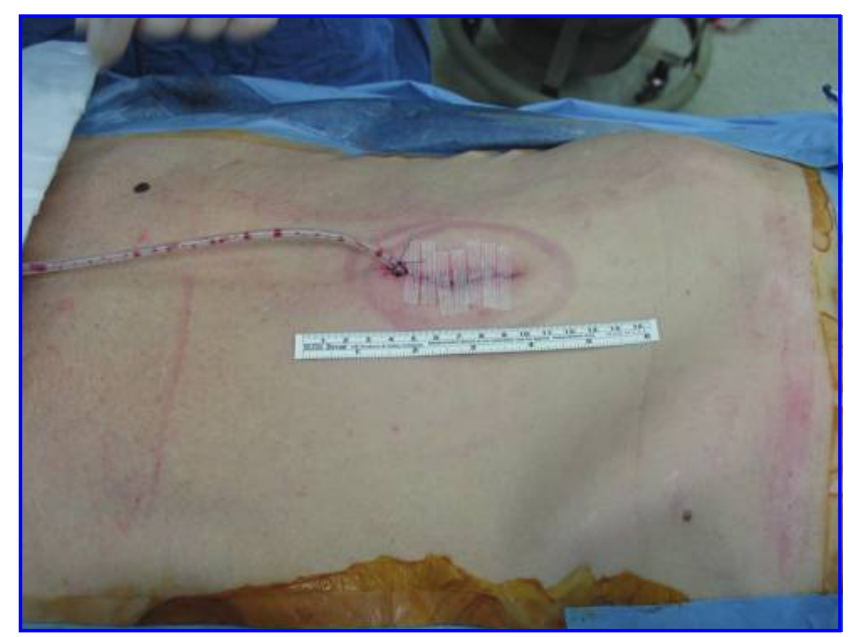

FIG. 4. Postoperative view of the wound.
Table 1. Perioperative Data of the Patients

\begin{tabular}{lcc}
\hline & Patient 1 & Patient 2 \\
\hline Age (years) & 56 & 68 \\
Sex & Female & Male \\
Body mass index $\left(\mathrm{kg} / \mathrm{m}^{2}\right)$ & 21.4 & 20.7 \\
Previous operation & - & - \\
Incision length (cm) & 4 & 4.5 \\
Total operative time (minutes) & 385 & 285 \\
$\quad$ Nephrectomy & 145 & 175 \\
Bladder cuff excision & 240 & 110 \\
Estimated blood loss (mL) & 100 & 350 \\
Pain score (VAS) & & \\
$\quad$ At 1 day & 6 & 5 \\
At 3 days & 2 & 2 \\
Hospital stay (days) & 3 & 3 \\
Foley catheter removal (days) & 3 & 7 \\
Complications & - & - \\
\hline
\end{tabular}

VAS $=$ visual analog scale.

\section{Discussion}

During the past several decades, minimally invasive surgery, including conventional laparoscopic surgery or LESS, has been used increasingly in the management of various urologic diseases. The goal of conventional laparoscopic surgery or LESS is to reproduce open surgical techniques in a minimally invasive manner. Because LESS is a novel technique, however, there are few literary reports about its application in malignancy.

There have been several reports that mention LESS nephroureterectomy for upper urinary tract TCC. ${ }^{3-5}$ They only reported, however, the lists and surgical outcomes of the various LESS procedures they performed ${ }^{3,4}$ or their procedure for the distal ureter is either not well described or done in an open fashion, which maintains the advantages of our study. To our knowledge, this is the first published report concerning the technical details of LESS nephroureterectomy with bladder cuff excision for the management of upper urinary tract TCC. These preliminary data demonstrate that LESS nephroureterectomy with bladder cuff excision is feasible,

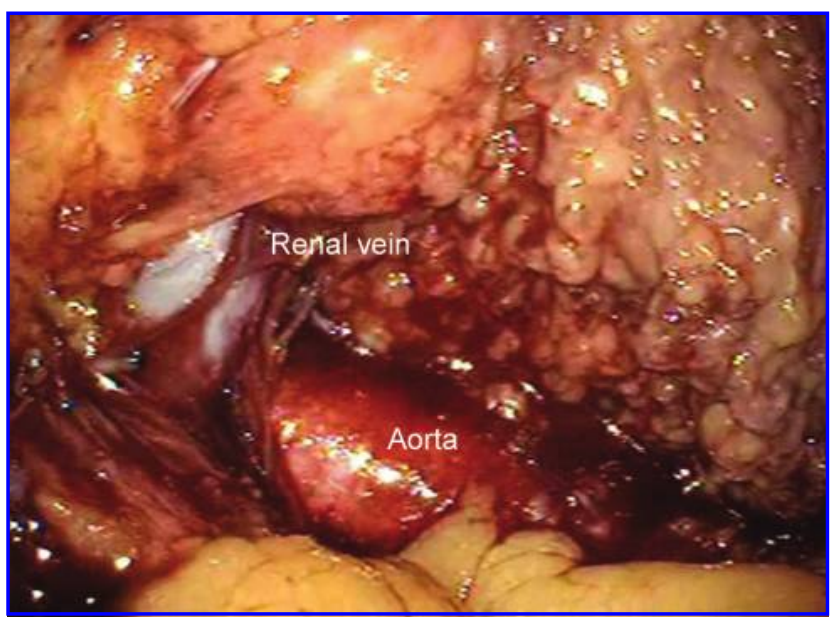

FIG. 5. Intraoperative view after lymph node dissection. 
safe, and based on the same oncologic principles as open surgical technique.

The principle of surgical treatment for upper urinary tract TCC is nephroureterectomy with en bloc excision of bladder cuff. Standard oncologic protocol necessitates resection of a 1$\mathrm{cm}$ bladder cuff around the ipsilateral ureteral orifice to excise and completely remove the distal intravesical ureter. There are still concerns, however, regarding the optimal technique of bladder cuff excision. In an attempt to adhere to these oncologic principles, a variety of techniques have been used when performing distal ureterectomy and bladder cuff excision, including cystoscopic resection of the ureteral orifice (pluck technique) ${ }^{7}$ cystoscopic detachment and ligation technique, ${ }^{8}$ laparoscopic stapling of the bladder cuff, ${ }^{9}$ and standard open technique.

Each technique has unique advantages and disadvantages. ${ }^{10,11}$ From an oncologic point of view, however, the open technique remains one of the most reliable and sound procedures. The advantages of the open technique are its familiarity to surgeons and adherence to the oncologic principles of managing upper urinary tract TCC. The risk of tumor extravasation is minimized, direct visual confirmation of a resected ureteral orifice decreases the likelihood of incomplete removal of the distal ureter, and the bladder opening can be adequately closed. In this regard, we concluded that our technique for LESS nephroureterectomy with bladder cuff excision essentially parallels the advantages of standard open techniques.

\section{Conclusion}

We described our surgical details for LESS nephroureterectomy with bladder cuff excision for the management of upper urinary tract TCC through a single umbilical incision. We concluded that LESS nephroureterectomy is a minimally invasive technique that may reproduce the open surgical technique and adhere to the same oncologic principles of open surgical technique.

\section{Disclosure Statement}

No competing financial interests exist.

\section{References}

1. Clayman RV, Kavoussi LR, Figenshau RS, et al. Laparoscopic nephroureterectomy: Initial clinical case report. J Laparoendosc Surg 1991;1:343-349.
2. Stolzenburg JU, Kallidonis $\mathrm{P}$, Till $\mathrm{H}$, et al. Current status of laparoendoscopic single-site surgery in urology. World J Urol 2009;Aug 1. Epub ahead of print.

3. Desai MM, Berger AK, Brandina R, et al. Laparoendoscopic single-site surgery: Initial hundred patients. Urology 2009; 74:805-812.

4. White WM, Haber GP, Goel RK, et al. Single-port urological surgery: Single-center experience with the first 100 cases. Urology 2009;74:801-804.

5. Ponsky LE, Steinway ML, Lengu IJ, et al. A Pfannenstiel single-site nephrectomy and nephroureterectomy: A practical application of laparoendoscopic single-site surgery. Urology 2009;74:482-485.

6. Park YH, Kang MY, Jeong MS, et al. Laparoendoscopic single-site nephrectomy using a homemade single-port device for single-system ectopic ureter in a child: Initial case report. J Endourol 2009;23:833-835.

7. McDonald HP, Upchurch WE, Sturdevant CE. Nephroureterectomy: A new technique. J Urol 1952;67:804-809.

8. Gill IS, Soble JJ, Miller SD, Sung GT. A novel technique for management of the en bloc bladder cuff and distal ureter during laparoscopic nephroureterectomy. J Urol 1999;161: $430-434$.

9. Shalhav AL, Dunn MD, Portis AJ, et al. Laparoscopic nephroureterectomy for upper tract transitional cell cancer: The Washington University experience. J Urol 2000;163: 1100-1104.

10. El Fettouh HA, Rassweiler JJ, Schulze M, et al. Laparoscopic radical nephroureterectomy: Results of an international multicenter study. Eur Urol 2002;42:447-452.

11. Brown JA, Strup SE, Chenven E, et al. Hand-assisted laparoscopic nephroureterectomy: Analysis of distal ureterectomy technique, margin status, and surgical outcomes. Urology 2005;66:1192-1196.

Address correspondence to: Hyeon Hoe Kim, M.D., Ph.D. Department of Urology Seoul National University Hospital 28 Yeongeon-dong, Jongno-gu Seoul 110-744

Korea

E-mail: hhkim@snu.ac.kr

\section{Abbreviations Used}

$\mathrm{BMI}=$ body mass index

LESS = laparoendoscopic single-site

$\mathrm{TCC}=$ transitional-cell carcinoma 


\section{This article has been cited by:}

1. Joo Yong Lee, Sang Jin Kim, Hong Sang Moon, Yong Tae Kim, Tchun Yong Lee, Sung Yul Park . 2011. Initial Experience of Laparoendoscopic Single-site Nephroureterectomy with Bladder Cuff Excision for Upper Urinary Tract Urothelial Carcinoma Performed by a Single Surgeon. Journal of Endourology 25:11, 1763-1768. [Abstract] [Full Text HTML] [Full Text PDF] [Full Text PDF with Links]

2. Francesco Greco, Luca Cindolo, Riccardo Autorino, Salvatore Micali, Robert J. Stein, Giampaolo Bianchi, Caterina Fanizza, Luigi Schips, Paolo Fornara, Jihad Kaouk. 2011. Laparoendoscopic Single-Site Upper Urinary Tract Surgery: Assessment of Postoperative Complications and Analysis of Risk Factors. European Urology . [CrossRef]

3. Kyung Hwa Choi, Won Sik Ham, Koon Ho Rha, Jae Won Lee, Hwang Gyun Jeon, Francis Raymond P. Arkoncel, Seung Choul Yang, Woong Kyu Han. 2011. Laparoendoscopic Single-Site Surgeries: A Single-Center Experience of 171 Consecutive Cases. Korean Journal of Urology 52:1, 31. [CrossRef]

4. Seok Young Lee, Yong Tae Kim, Hae Young Park, Tchun Yong Lee, Sung Yul Park. 2010. Initial Experience with Laparoendoscopic Single-Site Surgery by Use of a Homemade Transumbilical Port in Urology. Korean Journal of Urology 51:9, 613. [CrossRef] 